\title{
Comparison of SMOS and Aquarius Sea Surface Salinity and analysis of possible causes for the differences
}

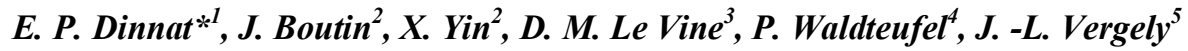 \\ ${ }^{1}$ Cryospheric Sciences Laboratory, NASA-GSFC and Chapman University, Greenbelt, MD, United States \\ ${ }^{2}$ Laboratoire d'Océanographie et du Climat: Expérimentations et Approches Numériques, CNRS/IRD/UPMC/MN, Paris, \\ France \\ ${ }^{3}$ Cryospheric Sciences Laboratory, NASA-GSFC, Greenbelt, MD, United States \\ ${ }^{4}$ Laboratoire Atmosphères, Milieux, Observations Spatiales, CNRS/UVSQ/UPMC, Paris, France
}

${ }^{5}$ ACRI-ST, 260 route du Pin Montard, Sophia Antipolis, France. ACRI-ST, 260 route du Pin Montard, Sophia Antipolis, France.

\section{Introduction}

Two ongoing space missions share the scientific objective of mapping the global Sea Surface Salinity (SSS), yet their observations show significant discrepancies. ESA's Soil Moisture and Ocean Salinity (SMOS) and NASA's Aquarius use L-band $(1.4 \mathrm{GHz})$ radiometers to measure emission from the sea surface and retrieve SSS. Significant differences in SSS retrieved by both sensors are observed, with SMOS SSS being generally lower than Aquarius SSS, except for very cold waters where SMOS SSS is the highest overall. Figure 1 is an example of the difference between the SSS retrieved by SMOS and Aquarius averaged over one month and 1 degree in longitude and latitude. Differences are mostly between -1 psu and +1 psu (psu, practical salinity unit), with a significant regional and latitudinal dependence. We investigate the impact of the vicarious calibration and some components of the retrieval algorithm used by both mission on these differences.

\section{Differences in SMOS and Aquarius algorithms}

One notable difference between the two missions' algorithms is the dielectric constant model used for the sea water. SMOS uses the model of Klein and Swift (1977) [1] and Aquarius uses the model of Meissner and Wentz (2012) [2]. Although similar, the two models are noticeably different, especially in cold water (Fig. 2, left). The dielectric constant model is used at two stages of the data processing: 1/ to calibrate the instruments by comparing radiometric measurements to forward model simulations, and 2/ to invert SSS from surface brightness temperature (Tb). In order to assess the impact of the dielectric constant model on the differences observed in SSS between SMOS and Aquarius, we reprocess the Aquarius data using the model used for SMOS. Specifically, we use the Klein and Swift model for the reference ocean used in the calibration of Aquarius; then we use it again, keeping all other factors the same, to perform the inversion to obtain SSS.

Another difference between the two missions concerns the vicarious calibration. SMOS Ocean Target Transformation (OTT) uses comparisons between measured Tb's and forward model simulations over a limited region in the Pacific Ocean to remove biases in its field of view [3]. Aquarius performs a similar comparison at global scale [4] . The reference SSS for the simulations is the World Ocean Atlas (2009) for SMOS [5], the HYbrid Coordinates Ocean Model (HYCOM) for Aquarius [6]. We assess the difference in the reference salinity fields that are used to calibrate both instruments.

Finally, the correction for Faraday rotation is performed using different approaches for both missions. Faraday rotation results in mixing up the vertical (V-pol) and horizontal (H-pol) polarizations of $\mathrm{Tb}$. It needs to be accounted for before retrieving SSS. The Faraday rotation angle is a function of the vertical Total Electron Content (TEC) up to the spacecraft altitude, the magnetic field vector $B$ and the geometry between $B$ and the sensor's line of sight. Aquarius retrieves the Faraday rotation angle using a combination of the measured third Stokes parameter and $\mathrm{Tb}$ at $\mathrm{V}$ - and H-pol as proposed in [7]. For now, SMOS algorithm retrieves a TEC value from SMOS data assuming TEC is constant over a dwell line and considering a prior value of TEC from the International GPS Service (IGS) data [8]; the Faraday rotation angle is then derived from this retrieved TEC and $B$ from the International Geomagnetic Reference Field (IGRF) [9][10]. However, a new technique for retrieving TEC from the third Stokes parameter measured by SMOS at high incidence angles has been developed [11]. We show some results from this new TEC estimates, and compare them with the IGS model. We also present the TEC derived from the Faraday angle retrieved by Aquarius and $B$ from the IGRF model. It should be noted that the IGS TEC reported here are always scaled to the spacecraft altitude. 


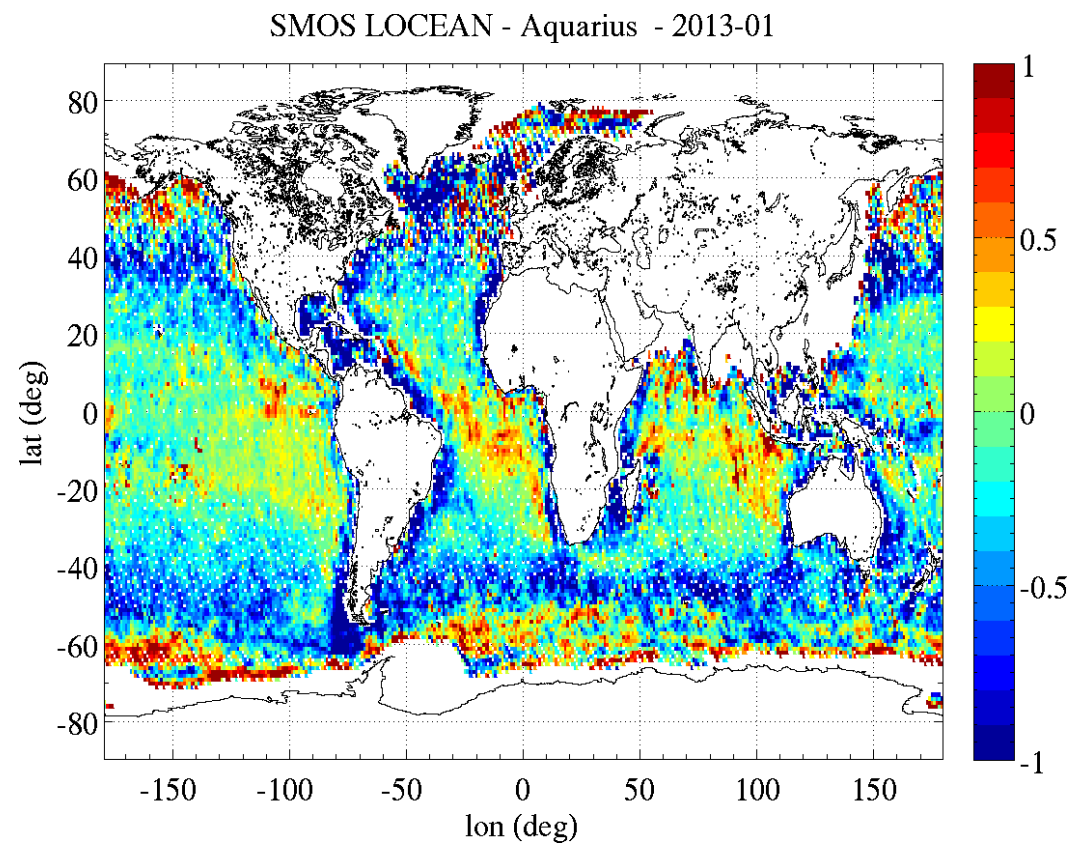

Figure 1: Global map of the difference in SSS between SMOS Level 3 (LOCEAN) product and Aquarius Level 2 product binned monthly at $1 \mathrm{deg} \times 1 \mathrm{deg}$ spatial resolution, for the month of January 2013. The colorscale is saturated between -1 psu and +1 psu.

\section{Results}

The two dielectric constant models exhibit differences of less than a percent, but this uncertainty results in differences in $\mathrm{Tb}$ of the order of a few tenths of a Kelvin (Fig. 2, left). The differences between Aquarius original data and data reprocessed with SMOS permittivity model vary mostly within $0.5 \mathrm{psu}$ at global scale, with a few larger regional variations, for example in cold waters (Fig. 2, right). Seasonal variations occur at mid and high latitudes. Aquarius and SMOS differences exhibit dependence in temperature (Fig. 1), which is reduced when Aquarius data are reprocessed with SMOS's permittivity model. We find that the permittivity model explains part of the differences between both instruments, particularly in cold waters, but some significant disagreement remains.

The differences in the reference SSS field are most of the time relatively small, but not always negligible (Fig. 3, left). Large regions of the ocean exhibit differences of just $0.1 \mathrm{psu}$ or less. However, regionally, differences can be larger ( 1 psu or more) and are variable in time. The difference in the region used for SMOS calibration varies between -0.1 psu and +0.05 psu since Aquarius started operating (Fig. 3, right).

A comparison between the TEC obtained from Aquarius measurement and the IGS model for May 2012 (Fig. 4, left) shows good qualitative agreement in general, but a systematic higher IGS TEC for the southern latitudes. For the high southern latitudes (higher than -40 degrees), Aquarius TEC is close to null, contrary to what IGS predicts. A comparison of SMOS retrieved TEC in May 2011 (Fig. 4, right) shows very similar results, with IGS showing a much larger TEC than the one retrieved from the SMOS measurements. Preliminary tests indicates that using a TEC derived from $\mathrm{Tb}$ measurements improve SMOS SSS retrieval ([11] and [12], this meeting), and should make Aquarius and SMOS more consistent with each other. 

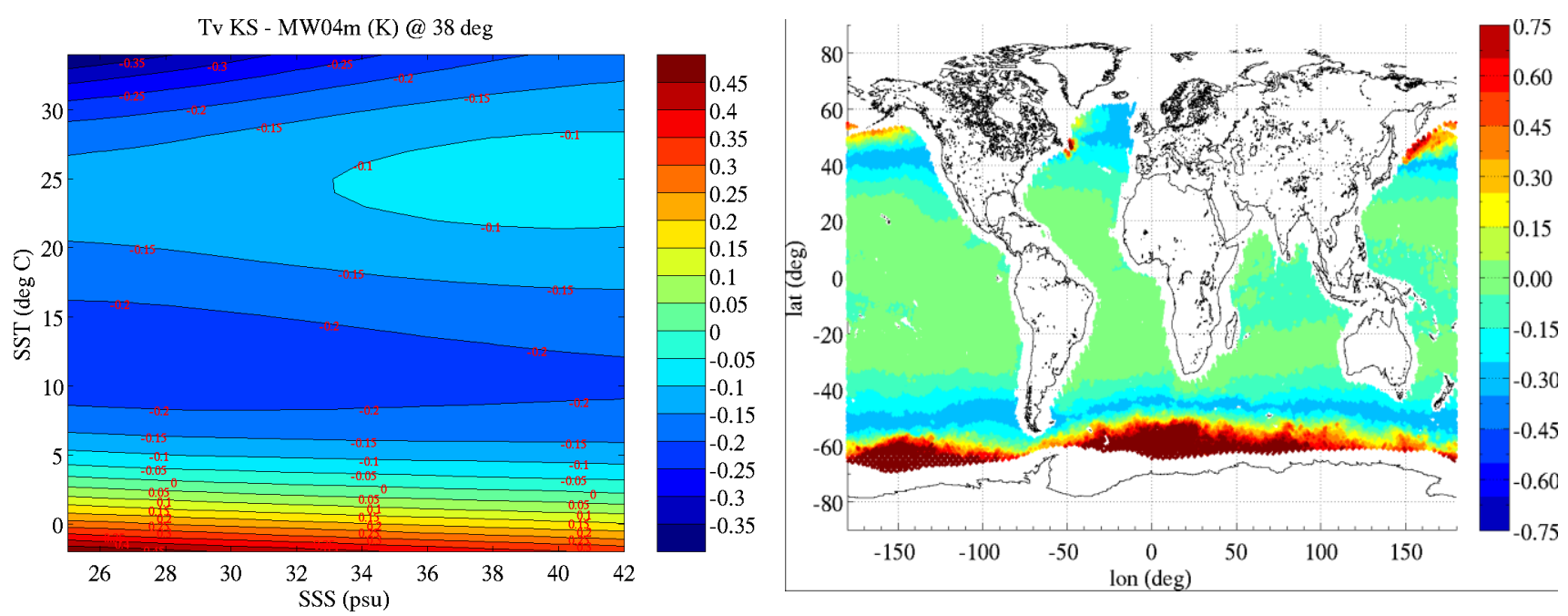

Figure 2: (left) Tb difference at vertical polarization for a smooth sea surface (i.e. Fresnel reflectivity) caused by differences in sea water dielectric constant model, computed between KS77 [1] and MW12 [2] models, versus Sea Surface Salinity (SSS) in psu and Sea Surface Temperature (SST) in Celsius. The incidence angle is 38 degrees. (right) Global map of the difference in Aquarius SSS (psu) for one week in early February 2012 caused by differences in dielectric constant models. The difference is between our reprocessed Aquarius data and the official Level 2 product. We compute the reprocessed data using the KS77 model for the calibration and the inversion of the Level 2 data into SSS. The official product uses MW12 for the calibration and inversion.

\section{Conclusion}

We assess the impact of the difference in dielectric constant model and reference salinity field on the difference in retrieved SSS between SMOS and Aquarius. We find that the dielectric constant has a large impact mostly in cold waters. Differences in reference SSS fields are small in general, but could explain biases of 0.1 psu at times. This research is ongoing and will address the differences in reference fields for the sea surface temperature. We also analyze the results of a new technique used to derive the Total Electron Content (TEC) from the third Stokes parameter measured by SMOS. Results appear consistent with the TEC derived from Aquarius measurements (although these preliminary tests were conducted for the same month but for different years) and lead to improved SMOS SSS retrieval ([11] and [12], this meeting) . Ultimately, a processing similar to SMOS will be applied to Aquarius data to assess the impact on SSS retrieval of several of the differences in the two instrument's algorithms.
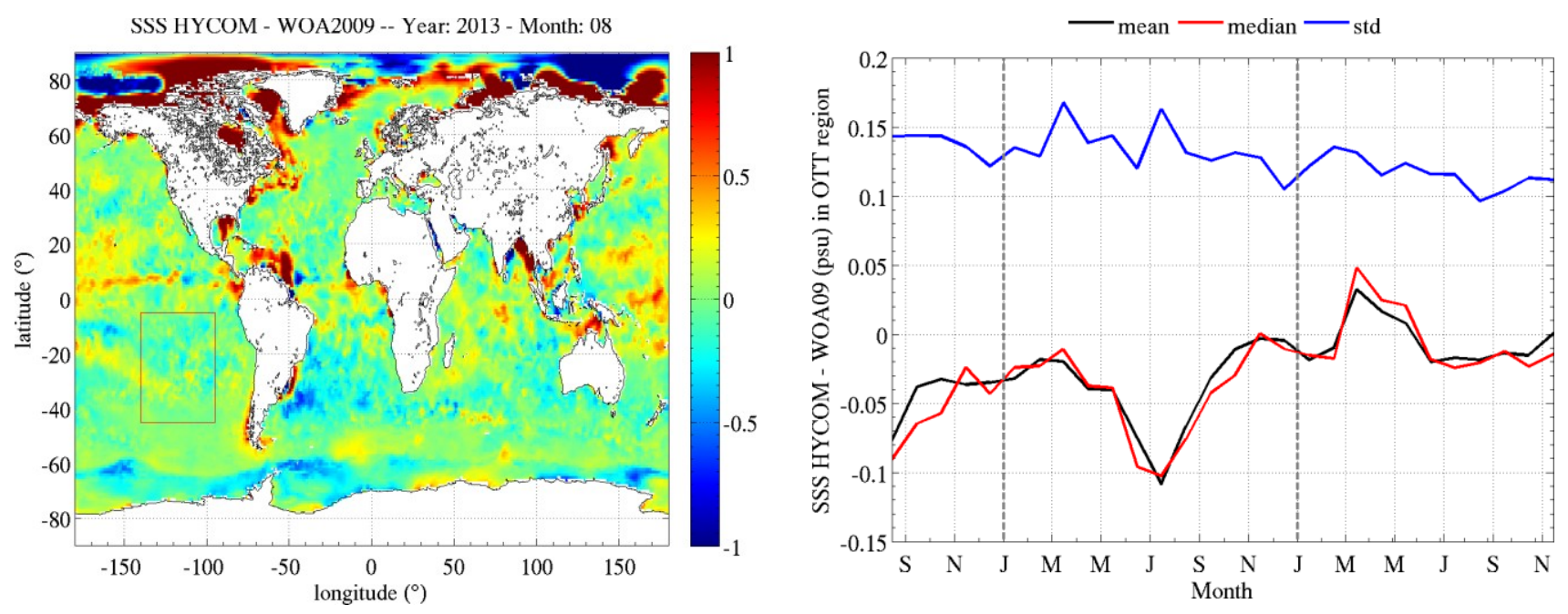

Figure 3: (left) Global monthly map of the differences in SSS (psu) between the two different ancillary products used in the calibration of Aquarius and SMOS. The difference is between the HYCOM model (used for Aquarius) and the World Ocean Atlas 2009 (used for SMOS). The red square in the south of the Pacific Ocean off the coast of South America illustrates the region used for the calibration of SMOS SSS product (i.e. the Ocean Target Transformation). (right) Time series of the average (mean and median) and standard deviation of the difference in SSS between HYCOM and WOA09 over the Ocean Target Transformation (OTT) region since the start of the Aquarius mission (Aug 2011 - Nov 2013). The vertical dashed lines part the different years. 


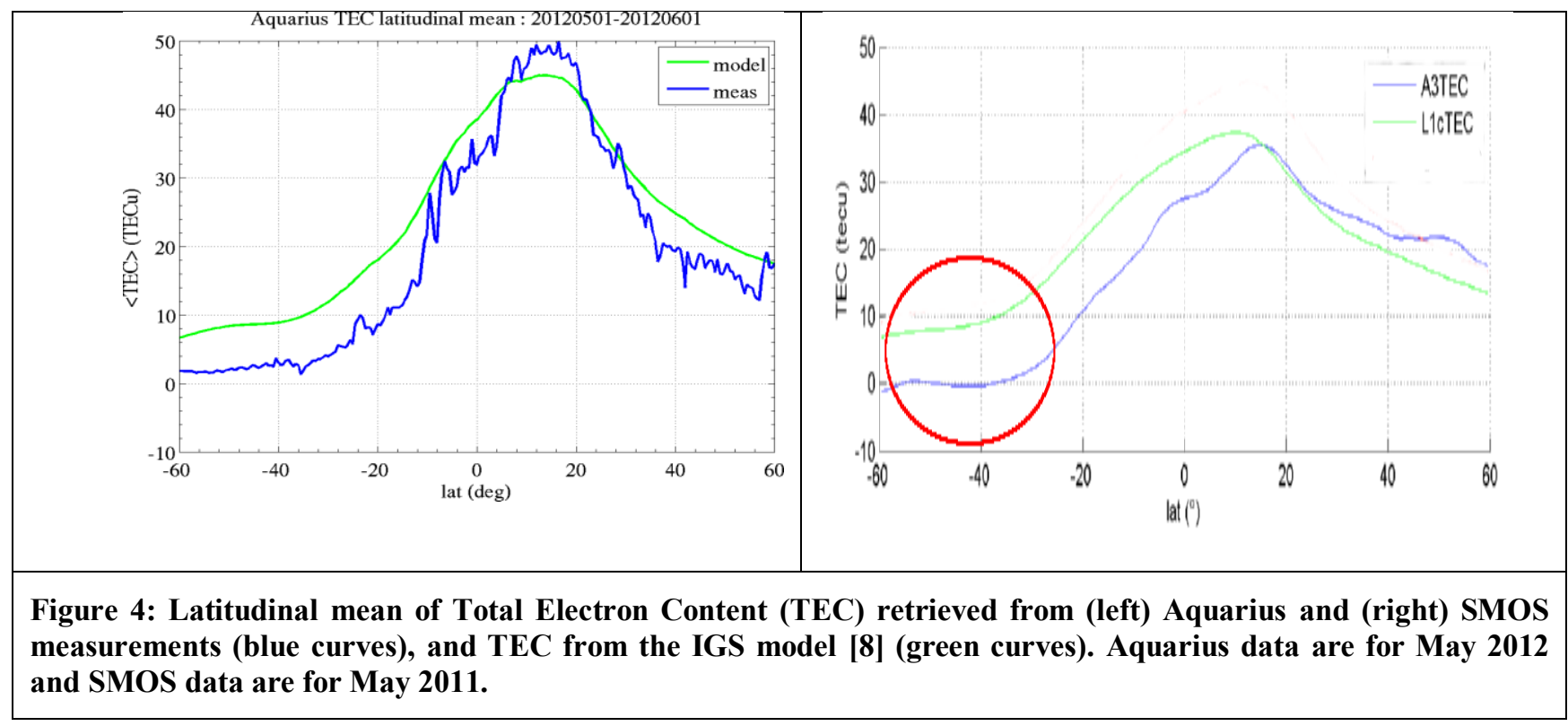

\section{References}

[1] L. A. Klein and C. T. Swift, "An improved model for the dielectric constant of sea water at microwave frequencies," IEEE Transactions on Antennas and Propagation, vol. AP-25, no. 1, pp. 104-111, 1977.

[2] T. Meissner and F. J. Wentz 2012, "The Emissivity of the Ocean Surface Between 6 and $90 \mathrm{GHz}$ Over a Large Range of Wind Speeds and Earth Incidence Angles", IEEE Trans. Geosci. Remote Sens., vol. 50, no. 8, pp. 30043026, Aug. 2012.

[3] X. Yin, J. Boutin and P. Spurgeon, "Biases Between Measured and Simulated SMOS Brightness Temperatures Over Ocean: Influence of Sun," Selected Topics in Applied Earth Observations and Remote Sensing, IEEE Journal of , vol.6, no.3, pp.1341-1350, June 2013.

[4] J. Piepmeier et al., "Aquarius radiometer post-launch calibration for product version 2," Aquarius Project Document: AQ-014-PS-0015, Tech. Rep. AQ-014-PS-0015, NASA and CONAE, 2013.

[5] J. Antonov et al., "World ocean atlas 2009 volume 2: Salinity," in NOAA Atlas NESDIS 69, S.Levitus, Ed. Washington, D.C., USA: U.S. Government Printing Office, p. 184, 2012.

[6] E. P. Chassignet et al., "The HYCOM (HYbrid Coordinate Ocean Model) data assimilative system," Journal of Marine Systems, vol. 65, Issues 1-4, pp. 60-83, March 2007.

[7] S. Yueh, "Estimates of Faraday rotation with passive microwave polarimetry for microwave remote sensing of Earth surfaces", IEEE Trans. Geosci. Remote Sens., 38(5), pp. 2434-2438, 2000.

[8] R. Crapolicchio, "VTEC Usage for the SMOS Level 1 Operational Processor (L1-OP)", ESA technical note XSMSGSEG-EOPG-TN-06-0019, 2008.

[9] C. C. Finlay et al., "International Geomagnetic Reference Field: the eleventh generation," International Association of Geomagnetism and Aeronomy, Working Group V-MOD, Geophys. J. Int., vol. 183, Issue 3, pp 1216-1230, December 2010.

[10] http://www.ngdc.noaa.gov/IAGA/vmod/igrf.html

[11] J.-L.Vergely, P. Waldteufel, J. Boutin, X. Yin, P. Spurgeon and S. Delwart, "Using the third Stokes parameter information for processing SMOS data", submitted to JGR-Ocean, 2014.

[12] X. Yin et al., "SMOS ocean salinity: recent improvements and applications", Proceedings of the XXXIst URSI General Assembly and Scientific Symposium, Beijing, China, August 17-23, 2014. 